\title{
Catalytic Hydrogenation and Hydrodeoxygenation of Furfural over Pt(111): A Model System for the Rational Design and Operation of Practical Biomass Conversion Catalysts
}

\author{
Martin J. Taylor, ${ }^{\dagger} \ddagger \odot$ Li Jiang, ${ }^{\S}$ Joachim Reichert, ${ }^{\S}$ Anthoula C. Papageorgiou, ${ }^{\S}$ Simon K. Beaumont, ${ }^{\| \odot}$
}

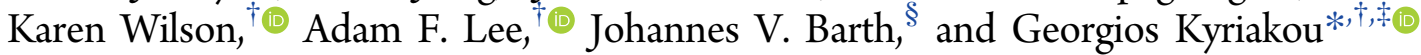

${ }^{\dagger}$ European Bioenergy Research Institute, Aston University, Aston Triangle, Birmingham B4 7ET, United Kingdom

${ }^{\ddagger}$ Chemical Engineering and Applied Chemistry, Aston University, Aston Triangle, Birmingham B4 7ET, United Kingdom

${ }^{\S}$ Physik-Department E20, Technische Universität München, D-85748 Garching, Germany

"Department of Chemistry, Durham University, South Road, Durham DH1 3LE, United Kingdom

\section{Supporting Information}

\begin{abstract}
Furfural is a key bioderived platform chemical whose reactivity under hydrogen atmospheres affords diverse chemical intermediates. Here, temperature-programmed reaction spectrometry and complementary scanning tunneling microscopy (STM) are employed to investigate furfural adsorption and reactivity over a $\mathrm{Pt}(111)$ model catalyst. Furfural decarbonylation to furan is highly sensitive to reaction conditions, in particular, surface crowding and associated changes in the adsorption geometry: furfural adopts a planar geometry on clean $\mathrm{Pt}(111)$ at low coverage, tilting at higher coverage to form a densely packed furfural adlayer. This switch in adsorption geometry strongly influences product selectivity. STM reveals the formation of hydrogen-bonded networks for planar furfural, which favor decarbonylation on clean Pt(111) and hydrogenolysis in the presence of coadsorbed hydrogen. Preadsorbed hydrogen promotes furfural hydrogenation to furfuryl alcohol and its subsequent hydrogenolysis to methyl furan, while suppressing residual surface carbon. Furfural chemistry over Pt is markedly different from that over $\mathrm{Pd}$, with weaker adsorption over the former affording a simpler product distribution than the latter; Pd catalyzes a wider range of chemistry, including ring-opening to form propene. Insight into the role of molecular orientation in controlling product selectivity will guide the design and operation of more selective and stable Pt catalysts for furfural hydrogenation.
\end{abstract}

\section{INTRODUCTION}

The development and sustainability of a bioresource-based chemical industry is strongly dependent on new heterogeneously catalyzed processes capable of selectively transforming bioderived organic molecules into valuable chemical intermediates, platform chemicals, and commercial products. In this respect, fundamental understanding of the underlying mechanistic aspects governing these heterogeneous processes is crucial. Lignocellulosic and oleochemical biomass-derived molecules are most attractive because of their abundance and the plethora of products that can be derived from them. Furfural is one of the most promising renewable platform compounds, ${ }^{1-4}$ itself obtainable via the acid-catalyzed hydrolysis of C5 sugars such as xylans and xylose.,

Furfural can be upgraded for a range of applications, finding use as a process agent for generating lubricating oils and in the flavorings and perfume industry where subtle structural alterations unlock diverse flavorings. ${ }^{3}$ However, the majority of furfural $(>62 \%)$ is hydrogenated to furfuryl alcohol for use as an adhesive, resin, or corrosion-resistant coating. ${ }^{2,6}$ Furfuryl alcohol is also a chemical building block to many other useful compounds in the fine chemical and pharmaceutical industries, as shown in Scheme $1,^{7}$ offering a route to 2-methyl furan, a potential biofuel, or tetrahydrofurfuryl alcohol (B) which can in turn undergo ring-opening hydrogenation to the polymer precursor 1,5-pentandiol (D). ${ }^{8}$ While 2-methyl furan production is reported for Pd hydrogenolysis catalysts in the liquid phase, ${ }^{9,10}$ it is observed only at high temperature over Pt. ${ }^{11-15}$

Large-scale furfural upgrading already occurs in China, South Africa, and the Dominican Republic, utilizing 280 000 tons of furfural per annum. ${ }^{1}$ However, current processes rely on a copper chromite catalyst which damages both ecologies and human health. ${ }^{16,17}$ This copper chromite catalyst operates at high pressure (up to 30 bar) and high temperature (up to 473

Received: February 23, 2017

Revised: $\quad$ March 28, 2017

Published: March 28, 2017 
Scheme 1. Furfural and Its Derivatives: (A)

Tetrahydrofuran, (B) Tetrahydrofurfuryl Alcohol, (C) 1,2Pentanediol, and (D) 1,5-Pentanediol

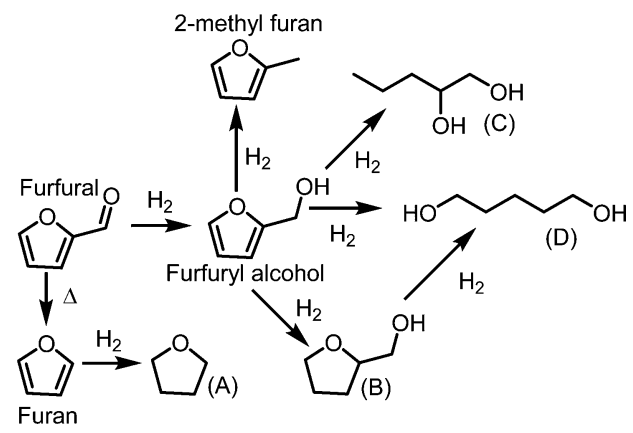

$\mathrm{K})$, affording acceptable selectivity and reasonable activity, but in light of its associated hazards, a replacement catalyst is urgently sought (especially if the transition from a petrochemical to biobased economy proceeds apace).,18

Furfural hydrogenation has been studied in both the vapor and liquid phase ${ }^{11,19-23}$ and is highly sensitive to reaction conditions over Pt catalysts. In general, liquid-phase hydrogenation is conducted at lower temperature than vapor-phase studies, hindering direct comparison because of the widely differing product distributions.

Higher temperature favors ring-opening or total hydrogenation of furfural, while mild conditions favor furfuryl alcohol and furan. ${ }^{11,20,24}$ Liquid-phase hydrogenation is complicated by poor hydrogen solubility in the reaction medium and competitive solvent coupling reactions to form $1^{\circ}$ and $2^{\circ}$ alcohols (prolific in furfural chemistry); ${ }^{11,12,14}$ hence, high hydrogen pressures are common. Vapor-phase studies require temperature only $>435 \mathrm{~K}$ to vaporize furfural at atmospheric pressure. $^{25}$ However, the key factors determining product selectivity and activity are not well-established. Many transitionmetal catalysts are reported for the hydrogenation of furfural to furfuryl alcohol, including $\mathrm{Ni}, \mathrm{Pd}, \mathrm{Co}, \mathrm{Cu}, \mathrm{Rh}, \mathrm{Ir}$, and $\mathrm{Ru}^{13,22,23,26-29}$ Platinum has been particularly explored under mild reaction conditions, ${ }^{11}$ for vapor-phase hydrogenation, with high reaction temperatures yielding a spectrum of small molecules including methyl furan..$^{20,30}$ In contrast, over $\mathrm{Pd}$, an array of products forms during liquid-phase furfural hydrogenation, ${ }^{10}$ such as acetals, ketals, and polymeric species, in addition to ring-opening products. ${ }^{31}$

Adsorption of unsaturated oxygenates over $\mathrm{Cu}, \mathrm{Ni}, \mathrm{Pd}$, and $\mathrm{Pt}(111)$ single-crystal surfaces and $\mathrm{Zn}$ adatom modified $\operatorname{Pt}(111)^{32-45}$ has been the focus of both experimental and theoretical investigations. For furfural, reactively formed furan (a decarbonylation product from furfural and furfuryl alcohol) behaves differently from molecular furan over $\operatorname{Pd}(111)$, the former being more prone to thermal decomposition to propylene. $^{39,46}$ Density functional theory (DFT) calculations have highlighted multiple reaction pathways for furfuryl alcohol hydrogenolysis over $\mathrm{Pd}(111)$ accompanied by the formation of adsorbed water; calculations suggest that the latter byproduct hinders furfural hydrogenation over $\mathrm{Cu}(111){ }^{32}$ Furfural adsorption and decomposition over $\mathrm{Pt}(111)$ and $\mathrm{Zn}$-modified $\operatorname{Pt}(111)$ have been extensively investigated using temperatureprogrammed desorption (TPD) and high-resolution electron energy loss spectroscopy by Shi and Vohs. ${ }^{38}$ They report that furfural adsorbs at low temperatures through the aromatic ring on $\mathrm{Pt}(111)$ driving unselective decomposition to $\mathrm{CO}$ and $\mathrm{H}_{2}$ upon heating. Surface modification with $\mathrm{Zn}$ adatoms favors furfural adsorption through the carbonyl carbon and associated ring tilting away from the $\mathrm{Pt}(111)$ surface. $^{38}$ This molecular reorientation suppresses thermal decomposition and ring hydrogenation in favor of hydrodeoxygenation (HDO) of the $\mathrm{C}=\mathrm{O}$ bond.

Here we utilize temperature-programmed reaction spectrometry (TPRS) and scanning tunneling microscopy (STM) to elucidate salient features of furfural's adsorption and hydrogenation over $\mathrm{Pt}(111)$ and correlate coverage-dependent orientation and self-assembly with selectivity toward hydrogenation versus decomposition pathways. Furfural adsorbs in a hydrogen-bonded planar network at low coverage, adopting a tilted geometry for a densely packed furfural adlayer. Adsorption geometry and hydrogen coadsorption influence selectivity toward both evolved products and surface carbon. Preadsorbed hydrogen promotes hydrogenation to furfuryl alcohol over decarbonylation to furan and passivates $\operatorname{Pt}(111)$ toward molecular decomposition. These results specifically allow us to discuss the fundamental surface behavior of furfural that leads to the selectivity of platinum versus other precious metal catalysts, such as palladium, in this important hydrogenation reaction. They also identify the critical importance of surface hydrogen concentration in both selectivity and deactivation as a result of surface coking by carbon. In consequence, the present findings help pave the way toward replacement catalysts for the undesirable copper chromite catalyst packages currently employed.

\section{EXPERIMENTAL SECTION}

TPRS was conducted in an ultrahigh vacuum (UHV) chamber operated at a base pressure of $3 \times 10^{-10}$ Torr, equipped with an Omicron 4 grid retarding field analyzer for low-energy electron diffraction (LEED) or Auger electron spectroscopy (AES) and a VG 300 quadrupole mass spectrometer whose ionizer was positioned $6 \mathrm{~mm}$ from the front face of the sample. The $\mathrm{Pt}(111)$ single crystal could be cooled to $140 \mathrm{~K}$ and resistively heated to above $1000 \mathrm{~K}$, monitored by a K-type thermocouple attached to the sample. Furfural (Sigma-Aldrich, 99\%), furan (Sigma-Aldrich, $\geq 99 \%$ ), 2-methyl furan (Sigma-Aldrich, 99\%), and furfuryl alcohol (Sigma-Aldrich, 98\%) were purified by several freeze-pump-thaw cycles. All organic molecules, $\mathrm{H}_{2}$ (Energas, 99.99\%), and $\mathrm{O}_{2}$ (Energas, 99.999\%) were delivered to the sample by backfilling the chamber to the required pressure. TPD and TPRS measurements were performed with a linear heating ramp of $9.9 \mathrm{~K} \mathrm{~s}^{-1}$. Selectivity calculations (see the Supporting Information) from the mass spectrometry data include corrections for mass spectrometer sensitivity and molecular ionization cross section achieved by admitting a known pressure of each molecule into the vacuum system, measuring the intensity of the mass fragments, and correcting the pressure gauge reading based on the theoretical ionization cross section. Mass fragments for molecular identification were as follows; furfural $(\mathrm{m} / z 96$ and 39), furan $(\mathrm{m} / z 68$ and 39), furfuryl alcohol $(\mathrm{m} / z 98,81$, and 39$)$, and methyl furan $(\mathrm{m} / z$ 82,53 , and 39 ). Additional ions were also monitored to identify potential products tetrahydrofuran $(\mathrm{m} / z 72)$, tetrahydrofurfuryl alcohol $(m / z 102)$, 2-methyltetrahydrofuran $(m / z 82)$, and propene $(m / z 42)$; however, none of these latter molecules were detected from furfural with and without coadsorbed $\mathrm{H}_{2}$. Exposures are quoted in Langmuirs (L), where $1 \mathrm{~L}$ is $1 \times 10^{-6}$ Torr s$^{-1}$ and have been corrected for ion gauge sensitivity. STM measurements were carried out in a separate custom built UHV 
system (base pressure low $10^{-10}$ Torr) with a commercial STM Aarhus 150 supplied by SPECS using a chemically etched tungsten tip held at $293 \mathrm{~K}$. Furfural molecules were dosed in situ. The tunneling bias $\left(V_{\mathrm{t}}\right)$ is applied to the sample. STM micrographs were processed using the WSxM software ${ }^{47}$ by adjustment of the plane and contrast and appropriate application of moderate Gaussian smoothing. Quoted coverages are based on estimation of the monolayer point (1 monolayer $=1 \mathrm{ML}$ ) from the appearance of a multilayer peak in the TPD data and of the highest density packing arrangement of molecules observed by STM in the high coverage image shown in Results and Discussion.

The single-crystal $\mathrm{Pt}(111)$ surfaces were cleaned by repeated cycles of $\mathrm{Ar}^{+}$(99.999\% Messer) sputtering $(1 \mathrm{keV}, 5 \mu \mathrm{A})$ for 40 min and annealing at $1000 \mathrm{~K}$. This process was followed by exposure to $5.5 \times 10^{-8}$ Torr $\mathrm{O}_{2}$ (Energas, $99.95 \%$ ) for $25 \mathrm{~min}$ at $815 \mathrm{~K}$. The sample was finally annealed to $1000 \mathrm{~K}$. Surface cleanliness was confirmed by LEED, AES, or STM.

\section{RESULTS AND DISCUSSION}

Adsorption and Reactivity of Furfural on $\mathrm{Pt}(111)$. Figure 1a shows TPRS acquired after clean $\mathrm{Pt}(111)$ was
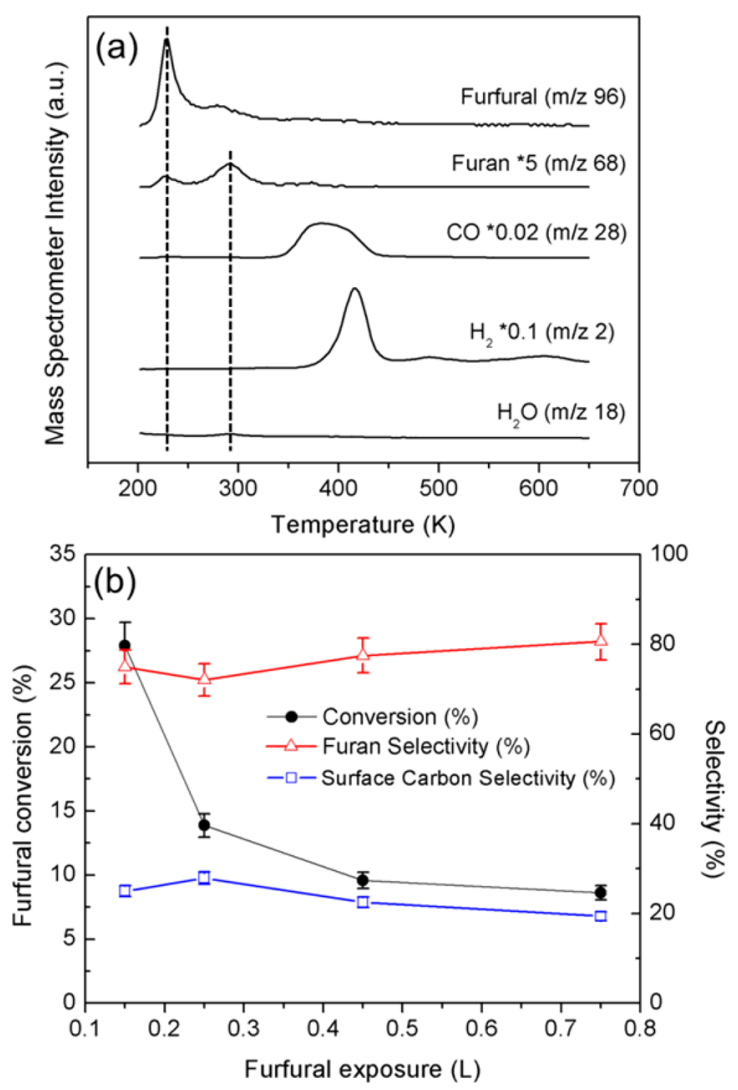

Figure 1. (a) Raw data showing the desorption products of furfural $(0.15 \mathrm{~L})$ on clean $\mathrm{Pt}(111)$. (b) Reactivity of the $\mathrm{Pt}(111)$ surface at varying furfural exposure.

exposed to $0.15 \mathrm{~L}$ furfural at $140 \mathrm{~K}$. The evolved products were unreacted furfural $(\mathrm{m} / z$ 96) and reactively formed furan $(\mathrm{m} / z$ 68), $\mathrm{CO}(m / z 28), \mathrm{H}_{2}(m / z 2)$, and $\mathrm{H}_{2} \mathrm{O}(\mathrm{m} / z 18)$. A total of $72 \%$ of the adsorbed furfural desorbed intact (main peak at 227 $\mathrm{K}$ with a smaller more strongly bound feature at $280 \mathrm{~K}$ ), whereas $\sim 22 \%$ reacted to furan which desorbed at $291 \mathrm{~K}$. The remainder formed surface carbon (see below), $\mathrm{CO}$, and $\mathrm{H}_{2}$.
The small feature at $280 \mathrm{~K}$ in the desorption spectrum of furfural is associated with desorption from step edges of the crystal, and it does not increase significantly in intensity, as shown in Figure S1a. Note that furfural multilayer peaks on $\mathrm{Pt}(111)$ appear at $190 \mathrm{~K}$ (Figure S1a,b). Note that the $227 \mathrm{~K}$ peak in the furan desorption corresponds to furfural, which shares a $m / z 68$ fragment with furan. This $m / z 68$ fragment is sufficiently weak that we can be confident it is not the main contributor to the furan desorption at $291 \mathrm{~K}$, and indeed the $280 \mathrm{~K}$ furfural peak is clearly offset in temperature from the former, confirming their different chemical origins. Carbon monoxide desorption due to furfural decarbonylation occurred at $384 \mathrm{~K}$, coincident with the temperature for chemisorbed $\mathrm{CO}$ desorption over $\mathrm{Pt}(111)$, indicating the former's appearance was desorption-rate limited; hence, decarbonylation occurs below $384 \mathrm{~K} . \mathrm{H}_{2}$ desorption from furfural decomposition occurred at 415,488 , and $604 \mathrm{~K}$. All three $\mathrm{H}_{2}$ desorption peak temperatures are higher than that of chemisorbed $\mathrm{H}_{2}$ over clean $\mathrm{Pt}(111)$; hence, their appearance was reaction-rate limited (see Figures 1a and S2a,b).

Liberation of surface atomic hydrogen from furfural decomposition (Figure 1a) does not lead to any selfhydrogenation products, presumably because the temperature for surface hydrogen formation is higher than the desorption temperature of furfuryl alcohol and methyl furan (Figure S3a,b). The hydrogen desorption peak area can be used to estimate the amount of residual carbon on $\mathrm{Pt}(111)$ following the temperature ramp as described in the Supporting Information. We estimate $\sim 6 \%$ of the total adsorbed furfural adlayer remains as carbonaceous deposits. Figure $1 \mathrm{~b}$ presents furfural total conversion and product selectivity as a function of exposure, revealing lower reactivity over crowded surfaces (conversion decreasing from $28 \%$ to $9 \%$ ), while furan selectivity (and the amount of residual carbon) were coverageindependent. This fall in furfural conversion and subsequent plateau occurs around $0.45 \mathrm{~L}$, coinciding with saturation of the furfural monolayer (Figure S1a). This saturation exposure is similar to that of $0.6 \mathrm{~L}$ reported by Shi and Vohs, ${ }^{38}$ with higher exposure rapidly populating multilayers.

A microscopic view of furfural on $\mathrm{Pt}(111)$ at two different coverages was obtained by STM (Figures $2 a-c$ and $3 a-c$ ).

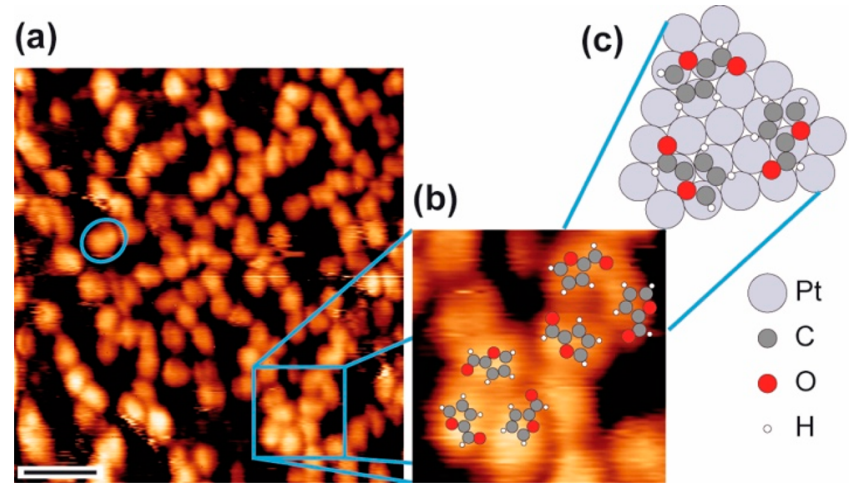

Figure 2. Submonolayer of furfural dosed to a $\mathrm{Pt}(111)$ surface kept at $95 \mathrm{~K}$. (a) Overview STM image with a single furfural molecule indicated by the blue circle $\left(T=145 \mathrm{~K}, V_{\mathrm{t}}=1.28 \mathrm{~V}, I_{\mathrm{t}}=0.12 \mathrm{nA}\right)$. The scale bar (black line) is $2 \mathrm{~nm}$. (b) Expanded area from panel a, indicated by the blue square, overlaid with an atomistic scale model with furfural in a planar geometry. (c) Model of proposed hydrogenbonded network on the $\mathrm{Pt}(111)$. 

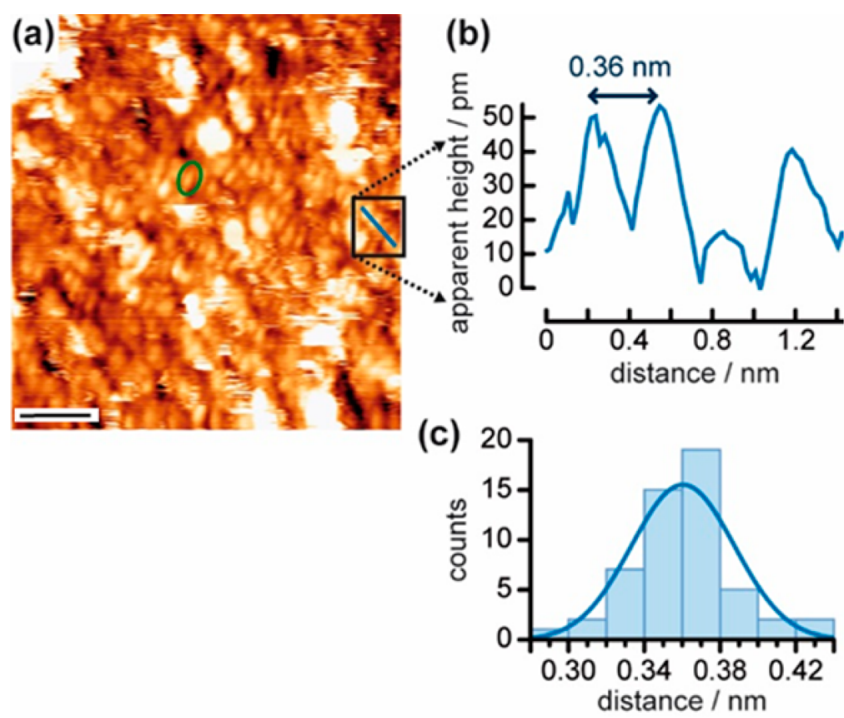

Figure 3. (a) STM image ( $\left.T=125 \mathrm{~K}, V_{\mathrm{t}}=-1.58 \mathrm{~V}, I_{\mathrm{t}}=0.16 \mathrm{nA}\right)$ with high furfural coverage dosed to a $\mathrm{Pt}(111)$ surface at $95 \mathrm{~K}$. At coverages without any bare $\mathrm{Pt}$, the line profile (b) across the line (highlighted by a square) indicated in panel $a$ in the same color shows that the molecular features (example outlined in purple) are separated by $\sim 0.36 \mathrm{~nm}$. The statistical separation of molecular features across the same direction is displayed in panel c. The scale bar (black line) in the STM image is $2 \mathrm{~nm}$.

Figure 2a shows a representative image for $\sim 0.5 \mathrm{ML}$ furfural, while Figure $3 a$ that of a saturated furfural adlayer, which also features some multilayer patches. Individual furfural molecules (outlined in blue) are observed to self-assemble in Figure 2a, presumably because of attractive adsorbate-adsorbate interactions. Considering the optimal furfural adsorption geometry calculated by DFT, ${ }^{36}$ we assign the bright protrusions in Figure $2 \mathrm{a}$ to individual furfural molecules. We further propose that this self-assembly is driven by weak hydrogen bonding between aromatic hydrogens and the carbonyl oxygen: aromatic $-\mathrm{C}-\mathrm{H} \cdots \mathrm{O}=\mathrm{C}-{ }^{48}$ as shown in Figure $2 \mathrm{~b}, \mathrm{c}$. At high coverage (Figure $3 \mathrm{a}$ ), these bright features change appearance, forming narrow protrusions (example outlined in green). Such protrusions can be found to be as close as $\sim 0.36 \mathrm{~nm}$ apart (Figure 3b), much closer than the molecular footprint of a planar furfural molecule. The statistical distribution of the molecular features across the same direction is centered $\sim 0.36$ $\mathrm{nm}$ (Figure 3c), which still cannot be accounted for with a model consisting of planar molecules. The packing density of furfural here is $\sim 3$ molecules $\mathrm{nm}^{-2}$ as compared to a maximum density of $\sim 2$ molecule $\mathrm{nm}^{-2}$ observed in the submonolayer surface (Figure 2a). This can be attributed to a change in the adsorption geometry toward a strongly tilted molecule, which is driven by the maximization of the number of molecules in contact with the metal surface.

At $245 \mathrm{~K}$ (above the main desorption peak of unreactive furfural), the STM reveals that at submonolayer coverages, some of the structures identified as single furfural molecules packing in the hydrogen-bonded networks are less discernible (Figure 4a). Smaller rounded protrusions enclosed within dotted blue circles whose dimensions are consistent with furan are now seen. At $265 \mathrm{~K}$, above the temperature for furfural desorption and around the onset of that for reactively formed furan desorption, Figure $4 \mathrm{~b}$ shows a decrease in the surface coverage of adsorbates.
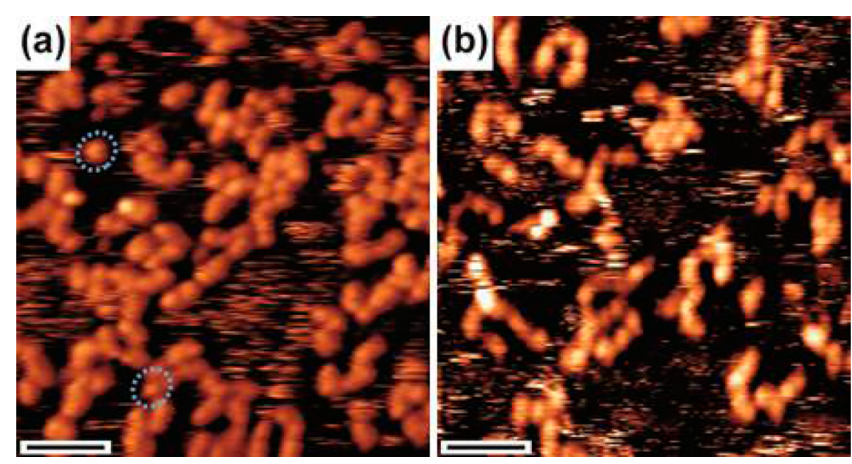

Figure 4. Temperature-dependent STM images of the molecular layer on $\mathrm{Pt}(111)$ after dosing $\sim 0.1 \mathrm{~L}$ furfural at $157 \mathrm{~K}$. (a) After the the sample is heated to $245 \mathrm{~K}$, molecular species consistent with furan, such as the ones in dotted circles, can be found $\left(V_{t}=1.06 \mathrm{~V}, I_{t}=0.10\right.$ $\mathrm{nA}$ ). (b) After further heating to $265 \mathrm{~K}$, some furan molecules have desorbed, reducing the surface coverage $\left(V_{\mathrm{t}}=-0.45 \mathrm{~V}, I_{\mathrm{t}}=0.07 \mathrm{nA}\right)$. The scale bar (black line) in the STM images is $2 \mathrm{~nm}$.

Desorption Enthalpies for Furfural and Furan on $\mathrm{Pt}$ (111). Enthalpies of furfural and furan desorption from $\mathrm{Pt}(111)$ were determined from a Redhead analysis, ${ }^{49}$ assuming a common pre-exponential factor of $10^{13} \mathrm{~s}^{-1}$ as widely adopted for organic adsorbates including phenol, ${ }^{50}$ benzene, ${ }^{51}$ and naphthalene. ${ }^{52}$ The desorption enthalpy of chemisorbed furfural in the main desorption peak at $227 \mathrm{~K}$ was $\sim 56$ $\mathrm{kJ} \mathrm{mol}^{-1}$ (Figure 1a). This value appears to be close to the value calculated for the desorption of phenol $\left(57 \mathrm{~kJ} \mathrm{~mol}^{-1}\right)$ and cyclopentane $\left(58 \mathrm{~kJ} \mathrm{~mol}^{-1}\right)$ on similar $\mathrm{Pt}(111)$ surfaces. $^{50,53}$ Furfural desorption from $\operatorname{Pd}(111)$ occurs at a far higher temperature $365 \mathrm{~K}^{39}$ and hence must be associated with a far greater activation barrier. Redhead analysis for reactively formed furan from flat-laying furan (Figure S4) reveals a desorption enthalpy of $\sim 73 \mathrm{~kJ} \mathrm{~mol}^{-1}$, close to that of unsubstituted aromatics such as benzene with $68 \mathrm{~kJ} \mathrm{~mol}^{-1}$ over $\operatorname{Pt}(111){ }^{51}$ DFT calculations for furfural on precious metals ${ }^{39,54,55}$ have produced different values depending on the metal used and are sensitive to the inclusion of dispersive interactions. ${ }^{54} \mathrm{~A}$ comparative study suggested that furfural adopts both $\mathrm{C}=\mathrm{C}$ ring and $\mathrm{C}=\mathrm{O}$ bonding motifs over $\mathrm{Pd}$, whereas on $\mathrm{Pt}$, adsorption occurs only through the $\mathrm{C}=\mathrm{C}$ ring. The latter adsorption geometry is consistent with the ability of furfural to form the hydrogen-bonded networks described above. The stronger adsorption of furfural over $\mathrm{Pd}(111)$ versus $\mathrm{Pt}(111)$ undoubtedly underpins their different reactivity: furfural evolves furan $(365 \mathrm{~K}), \mathrm{CO}(460 \mathrm{~K})$, and $\mathrm{H}_{2}(330$ and $410 \mathrm{~K})$ over $\operatorname{Pd}(111)$ but also undergoes extensive ring decomposition leading to propylene $(385 \mathrm{~K}) .^{37,39}$ In contrast, propylene was not observed over $\mathrm{Pt}(111)$ in this work.

Investigation and Quantification of Surface Carbon Product during Furfural Desorption from $\mathrm{Pt}(111)$. As discussed above, furfural adsorption over Pt results in carbon deposition, presenting a major technological drawback to utilizing Pt for large-scale furfural hydrogenation. Figure 5a,b shows consecutive TPRS profiles following a $0.25 \mathrm{~L}$ furfural exposure over $\mathrm{Pt}(111)$, without cleaning the surface each cycle. Figure 5a shows a $47 \%$ drop in furfural desorption intensity between the first and second cycle, with the amount of reactively formed hydrogen (Figure $5 b$ ) reduced by a similar amount (40\%). However, between the second and third exposure, the furfural and hydrogen desorption intensities decrease by only a further $5 \%$ and $3 \%$, respectively. These 

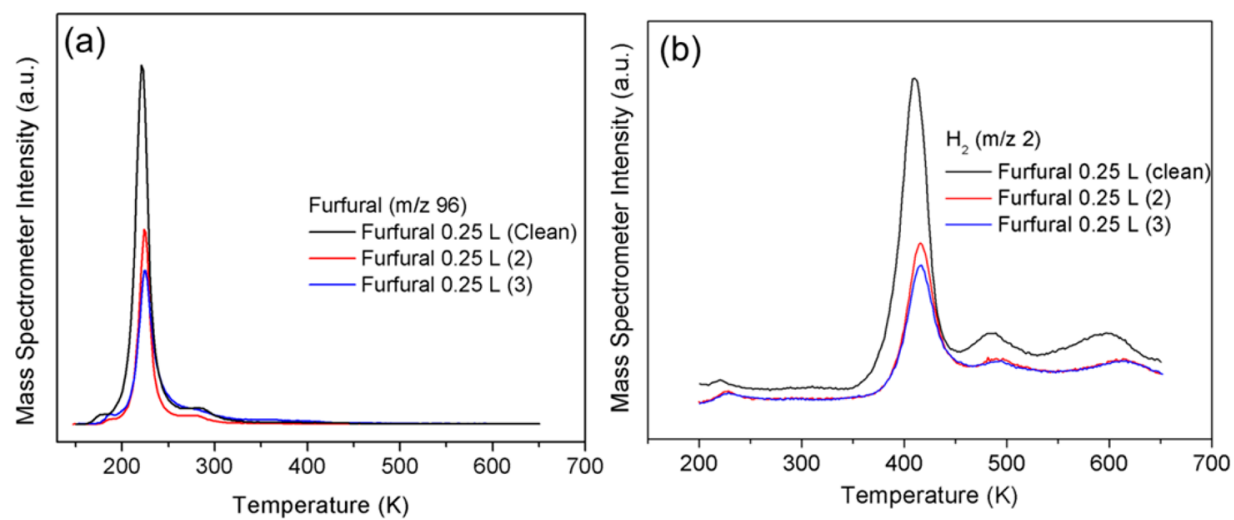

Figure 5. (a) Repeated exposure of furfural $(0.25 \mathrm{~L})$, followed by desorption, without surface cleaning in between cycles, showing diminished overall monolayer adsorption feature intensity at $222 \mathrm{~K}$, indicating site blocking by carbon deposits. (b) Corresponding decrease in $\mathrm{H}_{2}$ signal during adsorption-desorption cycles showing a decrease in reactive furfural due to carbonaceous deposits.
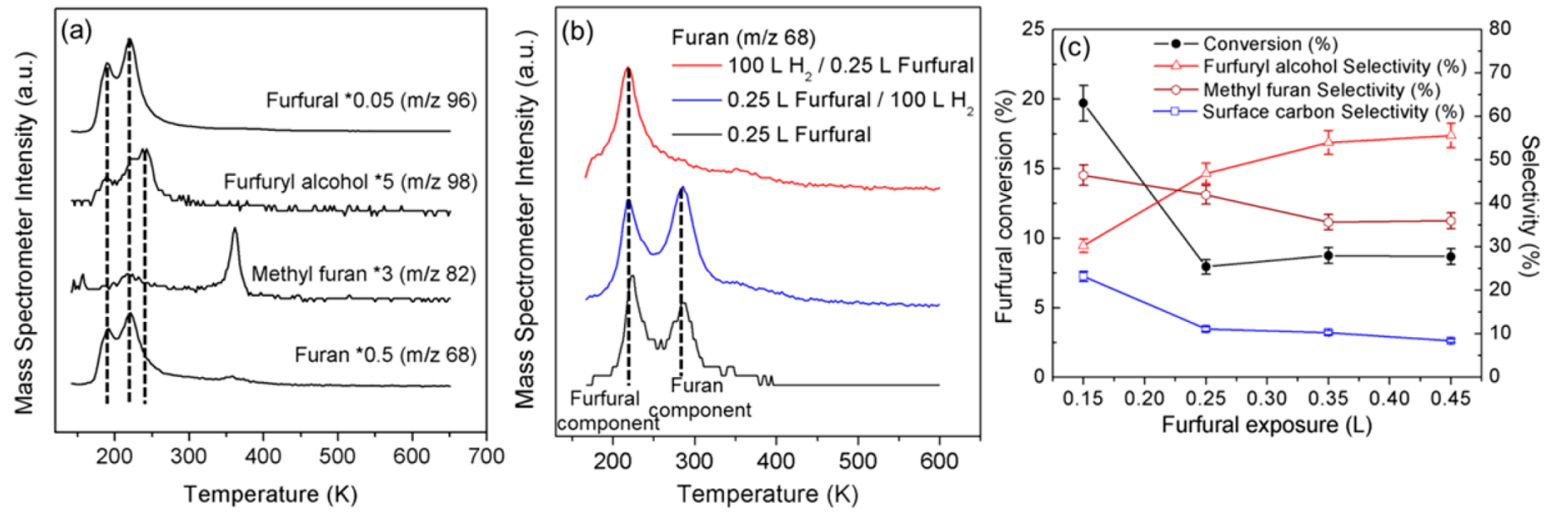

Figure 6. (a) TPRS for $\mathrm{Pt}(111)$ exposed to $100 \mathrm{~L} \mathrm{H}_{2}$ followed by $0.35 \mathrm{~L}$ furfural. (b) Reactively formed furan production from furfural over Pt(111) surfaces. (c) Furfural reactivity over $\mathrm{Pt}(111)$ pre-exposed to $100 \mathrm{~L} \mathrm{H}_{2}$ as a function of furfural exposure. Molecular adsorption was performed at 140 $\mathrm{K}$ in all cases.

observations demonstrate the number of available adsorption sites has decreased significantly, indicating the accumulation of significant (carbonaceous) residues. Furthermore, the initial decrease in furfural desorption of $\sim 47 \%$ between cycles one and two is significantly greater than the proportion of adsorbed furfural calculated to decompose to carbon $(\sim 20 \%$ from Figure $1 b)$. This suggests that any carbon deposits are likely uniformly distributed across the $\mathrm{Pt}(111)$ surface and hence block a large number of furfural adsorption sites. The formation of carbonaceous deposits from furfural is reportedly favored at 377-385 K over Pt catalysts during gas-phase hydrogenation, ${ }^{20,56}$ comparable to the desorption temperature for reactively formed hydrogen (indicative of hydrocarbon decomposition) from furfural over $\mathrm{Pt}(111)$ shown in Figure 1a. High furan yields during liquid-phase hydrogenation of furfural over Pt nanoparticles is also reported around $343 \mathrm{~K}^{11}$ in good agreement with that for the desorption of reactively formed furan from $\mathrm{Pt}(111)$ in this work at $323 \mathrm{~K}$. CO formed through furfural decarbonylation to furan may also be responsible for site-blocking and poisoning of Pt catalysts at temperatures below that necessary for desorption of the former. ${ }^{11}$ Poisoning by carbon laydown typically requires catalyst reactivation (e.g., through calcination) and concomitant loss in metal surface area or changes in particle morphology and hence is often considered irreversible. In contrast, reversible $\mathrm{CO}$ poisoning may be mitigated by higher-temperature operation.
Furfural Hydrogenation on Pt(111). Hydrogen adsorption over clean $\mathrm{Pt}(111)$ was first studied by TPRS (Figure S2b) as a function of exposure. The desorption temperature of molecular hydrogen decreased with increasing $\mathrm{H}_{2}$ exposure in accordance with the expected second-order kinetics reported by Gebhardt and Koel. ${ }^{57}$ Hydrogen coverages were calculated according to the work of Ertl and co-workers wherein $\theta \mathrm{H}_{\text {sat }}$ was $0.8 \mathrm{ML}^{58}$ Furfural and hydrogen were codosed, employing a $\mathrm{H}_{2}$ exposure of $100 \mathrm{~L}$ (corresponding to $\sim 0.4 \mathrm{ML}$ ) to ensure vacant $\mathrm{Pt}$ sites were available for furfural adsorption.

Figure 6a shows data from TPRS of a $100 \mathrm{~L} \mathrm{H}_{2}$ exposure and subsequent $0.35 \mathrm{~L}$ furfural exposure at $140 \mathrm{~K}$ over $\mathrm{Pt}(111)$. Furfural exhibits a multilayer desorption peak at $\sim 190 \mathrm{~K}$ and a monolayer desorption at $227 \mathrm{~K}$ (Figures $6 \mathrm{a}$ and S1b). Similar total yields of reactively formed furfuryl alcohol $(\mathrm{m} / z$ 98) and methyl furan $(\mathrm{m} / z \mathrm{82})$ were observed at 240 and $360 \mathrm{~K}$, respectively; however, no furan desorption was observed (Figure 6b). Note that furan, furfuryl alcohol, and methyl furan desorptions contain a contribution from furfural (which also exhibits mass fragments at $m / z 68,82$, and 98); however, the desorption temperatures of reactively formed furfuryl alcohol and methyl furan differ from that of furfural but are in close agreement to those observed from their respective molecularly adsorbed species (Figure S3a,b), indicating that their appearance is desorption-rate limited. Figure S2a shows the appearance of a low-temperature $\mathrm{H}_{2}$ desorption peak around $306 \mathrm{~K}$, indicated by an arrow, characteristic of the 
recombinative desorption of molecularly adsorbed hydrogen (Figure S2b). Additional hydrogen desorption must arise from the coadsorbed furfural and indeed are identical to those observed following furfural adsorption over clean $\mathrm{Pt}(111)$ at 415, 488, and $604 \mathrm{~K}$ (Figure 1a). As noted in an earlier section, furfural autohydrogenation over $\mathrm{Pt}(111)$ does not occur (Figure 1a); however, the observation of furfuryl alcohol in the presence of coadsorbed hydrogen indicates that furfural hydrogenation is possible over $\mathrm{Pt}(111)$ under UHV, provided that a high concentration of hydrogen adatoms is available at a relatively low surface temperature.

Reversing the dosing sequence, such that $\mathrm{Pt}(111)$ was first exposed to $0.25 \mathrm{~L}$ furfural followed by $100 \mathrm{~L} \mathrm{H}_{2}$, suppressed hydrogenation pathways to both furfuryl alcohol and methyl furan, affording a conversion and selectivity similar to that seen over clean $\mathrm{Pt}(111)$. The main product observed is furan desorbing at $291 \mathrm{~K}$ (Figure 6b). Figure 6c shows furfural conversion and selectivity as a function of furfural exposure over $\mathrm{Pt}(111)$ precovered with $100 \mathrm{~L} \mathrm{H}_{2}$. The results are in excellent agreement with the reaction pathways proposed in Scheme 1. At low furfural exposures (corresponding to a high ratio of surface $\mathrm{H}(\mathrm{a})$ :furfural), the stepwise hydrogenation of furfural to furfuryl alcohol, and its subsequent HDO to methyl furan, is favored. At high furfural exposures (a low surface $\mathrm{H}(\mathrm{a})$ :furfural ratio), insufficient surface hydrogen is available to further hydrogenate reactively formed furfuryl alcohol, which hence becomes the dominant product; however, in the latter scenario, more furfural desorbs molecularly.

The preceding insight enables us to predict the selectivity to furfuryl alcohol during furfural hydrogenation (Figure 7);

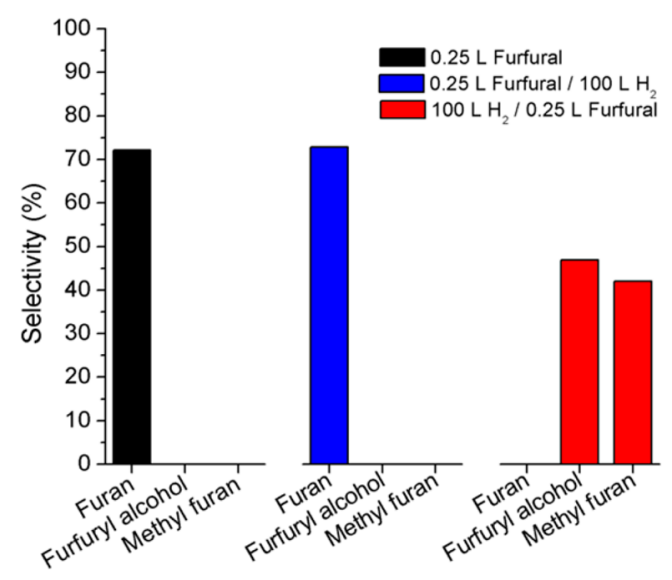

Figure 7. Gas-phase selectivities to molecular products for furfural with and without $\mathrm{H}_{2}$ and for different orders of exposure to the two reactants.

higher surface hydrogen concentrations encountered in gasphase furfural hydrogenation are expected to favor methyl furan relative to furfuryl alcohol, whereas the latter should be favored in the liquid phase. This is precisely as reported in the literature. ${ }^{11,12,20,59}$

\section{CONCLUSIONS}

The hydrogenation of furfural on $\mathrm{Pt}(111)$ was found to be highly sensitive to the conditions, in particular, surface crowding and associated changes in the adsorption geometry, used to carry out the catalytic reaction. On clean $\mathrm{Pt}(111)$, furfural adopts a planar motif at low coverages and a more tilted geometry as the coverage is increased. The extent of decarbonylation to furan was found to depend strongly on the coverage (and therefore adsorption geometry): at low coverage, the planar motif results in a much greater conversion to furan than occurs in the higher-coverage tilted molecules. The formation of surface carbon and possible consequences for practical catalyst deactivation processes have also been investigated and discussed.

Control of the furfural coverage on the surface is critical to the reaction selectivity, with hydrogenation and hydrogenolysis being possible at higher coverages. A strong correlation between the reactivity of the $\mathrm{Pt}(111)$ surface with that of $\mathrm{Pt}$ dispersed systems was observed, which enables the prediction of the activity and selectivity of Pt-based catalysts under practical conditions in the liquid and the gas phase. The order in which the surface encounters hydrogen and furfural is critically important. When furfural encounters a bare surface, it hinders the subsequent dissociative chemisorption of hydrogen and resulting hydrogenation activity. In contrast, when furfural encounters preadsorbed atomic hydrogen, furfuryl alcohol and methyl furan are produced; the former is a product of direct furfural hydrogenation, and the latter is a secondary product arising from the HDO of furfuryl alcohol (requiring a high ratio of surface $\mathrm{H}(\mathrm{a})$ :furfural). At a low surface $\mathrm{H}(\mathrm{a})$ :furfural ratio, insufficient surface hydrogen is available to further hydrogenate reactively formed furfuryl alcohol, which hence becomes the dominant product. Our results indicate that control over the furfural adsorption geometry, and surface hydrogen concentration, are key considerations for the design and operation of practical Pt catalysts for this important bioeconomy transformation.

\section{ASSOCIATED CONTENT}

\section{S Supporting Information}

The Supporting Information is available free of charge on the ACS Publications website at DOI: 10.1021/acs.jpcc.7b01744.

Additional TPR spectra as well as the protocols used for calculating reagent conversion and product selectivities (PDF)

\section{AUTHOR INFORMATION}

\section{Corresponding Author}

*E-mail: g.kyriakou@aston.ac.uk.

ORCID

Martin J. Taylor: 0000-0002-8810-8942

Anthoula C. Papageorgiou: 0000-0003-1054-0097

Simon K. Beaumont: 0000-0002-1973-9783

Karen Wilson: 0000-0003-4873-708X

Adam F. Lee: 0000-0002-2153-1391

Georgios Kyriakou: 0000-0001-6295-844X

Notes

The authors declare no competing financial interest.

\section{ACKNOWLEDGMENTS}

M.J.T. thanks Aston University for a Ph.D. studentship; K.W. thanks the EPSRC for funding under EP/K014676/1 and EP/ K014749/1; L.J. acknowledges funding of the China Scholarship Council (CSC). This work was supported by the European Research Council Advanced Grant MolArt (No. 247299). S.K.B. thanks both the Durham University Addison Wheeler scheme and the Leverhulme Trust's Early Career Fellowship 
scheme. G.K. acknowledges funding from the Royal Society and EPSRC (EP/M005186/2)

\section{REFERENCES}

(1) Yan, K.; Wu, G.; Lafleur, T.; Jarvis, C. Production, properties and catalytic hydrogenation of furfural to fuel additives and value-added chemicals. Renewable Sustainable Energy Rev. 2014, 38, 663-676.

(2) Mandalika, A.; Qin, L.; Sato, T. K.; Runge, T. Integrated biorefinery model based on production of furans using open-ended high yield processes. Green Chem. 2014, 16, 2480-2489.

(3) Gallezot, P. Conversion of biomass to selected chemical products. Chem. Soc. Rev. 2012, 41, 1538-1558.

(4) Climent, M. J.; Corma, A.; Iborra, S. Conversion of biomass platform molecules into fuel additives and liquid hydrocarbon fuels. Green Chem. 2014, 16, 516-547.

(5) Elliott, D. C.; Hart, T. R. Catalytic hydroprocessing of chemical models for bio-oil. Energy Fuels 2009, 23, 631-637.

(6) Besson, M.; Gallezot, P.; Pinel, C. Conversion of biomass into chemicals over metal Catalysts. Chem. Rev. 2014, 114, 1827-1870.

(7) Sharma, R. V.; Das, U.; Sammynaiken, R.; Dalai, A. K. Liquid phase chemo-selective catalytic hydrogenation of furfural to furfuryl alcohol. Appl. Catal., A 2013, 454, 127-136.

(8) Nakagawa, Y.; Tomishige, K. Production of 1,5-pentanediol from biomass via furfural and tetrahydrofurfuryl alcohol. Catal. Today 2012, 195, 136-143.

(9) Iqbal, S.; Liu, X.; Aldosari, O. F.; Miedziak, P. J.; Edwards, J. K.; Brett, G. L.; Akram, A.; King, G. M.; Davies, T. E.; Morgan, D. J.; et al. Conversion of furfuryl alcohol into 2-methylfuran at room temperature using $\mathrm{Pd} / \mathrm{TiO}_{2}$ catalyst. Catal. Sci. Technol. 2014, 4, 2280-2286.

(10) Aldosari, O. F.; Iqbal, S.; Miedziak, P. J.; Brett, G. L.; Jones, D. R.; Liu, X.; Edwards, J. K.; Morgan, D. J.; Knight, D. K.; Hutchings, G. J. Pd-Ru/ $\mathrm{TiO}_{2}$ catalyst - an active and selective catalyst for furfural hydrogenation. Catal. Sci. Technol. 2016, 6, 234-242.

(11) Taylor, M. J.; Durndell, L. J.; Isaacs, M. A.; Parlett, C. M. A.; Wilson, K.; Lee, A. F.; Kyriakou, G. Highly selective hydrogenation of furfural over supported Pt nanoparticles under mild conditions. Appl. Catal., B 2016, 180, 580-585.

(12) Chen, X.; Zhang, L.; Zhang, B.; Guo, X.; Mu, X. Highly selective hydrogenation of furfural to furfuryl alcohol over Pt nanoparticles supported on g- $\mathrm{C}_{3} \mathrm{~N}_{4}$ nanosheets catalysts in water. Sci. Rep. 2016, 6, 28558.

(13) Bhogeswararao, S.; Srinivas, D. Catalytic conversion of furfural to industrial chemicals over supported $\mathrm{Pt}$ and $\mathrm{Pd}$ catalysts. J. Catal. 2015, 327, 65-77.

(14) Merlo, A. B.; Vetere, V.; Ruggera, J. F.; Casella, M. L. Bimetallic $\mathrm{PtSn}$ catalyst for the selective hydrogenation of furfural to furfuryl alcohol in liquid-phase. Catal. Commun. 2009, 10, 1665-1669.

(15) Merlo, A. B.; Vetere, V.; Ramallo-López, J. M.; Requejo, F. G.; Casella, M. L. Liquid-phase furfural hydrogenation employing silicasupported $\mathrm{PtSn}$ and $\mathrm{PtGe}$ catalysts prepared using surface organometallic chemistry on metals techniques. React. Kinet., Mech. Catal. 2011, 104, 467-482.

(16) Egeblad, K.; Rass-Hansen, J.; Marsden, C.; Taarning, E.; Hviid Christensen, C. Heterogeneous catalysis for production of value-added chemicals from biomass. Catalysis 2009, 21, 13-50.

(17) Gowda, A. S.; Parkin, S.; Ladipo, F. T. Hydrogenation and hydrogenolysis of furfural and furfuryl alcohol catalyzed by ruthenium(II) bis(diimine) complexes. Appl. Organomet. Chem. 2012, 26, 86-93.

(18) Wojcik, B. H. Catalytic hydrogenation of furan compounds. Ind. Eng. Chem. 1948, 40, 210-216.

(19) Nagaraja, B. M.; Siva Kumar, V.; Shasikala, V.; Padmasri, A. H.; Sreedhar, B.; David Raju, B.; Rama Rao, K. S. A highly efficient $\mathrm{Cu}$ / $\mathrm{MgO}$ catalyst for vapour phase hydrogenation of furfural to furfuryl alcohol. Catal. Commun. 2003, 4, 287-293.

(20) Pushkarev, V. V.; Musselwhite, N.; An, K.; Alayoglu, S.; Somorjai, G. A. High structure sensitivity of vapor-phase furfural decarbonylation/hydrogenation reaction network as a function of size and shape of Pt nanoparticles. Nano Lett. 2012, 12, 5196-5201.
(21) Pang, S. H.; Schoenbaum, C. A.; Schwartz, D. K.; Medlin, W. J. Effects of thiol modifiers on the kinetics of furfural hydrogenation over Pd catalysts. ACS Catal. 2014, 4, 3123-3131.

(22) Villaverde, M. M.; Bertero, N. M.; Garetto, T. F.; Marchi, A. J. Selective liquid-phase hydrogenation of furfural to furfuryl alcohol over Cu-based catalysts. Catal. Today 2013, 213, 87-92.

(23) Audemar, M.; Ciotonea, C.; De Oliveira Vigier, K.; Royer, S.; Ungureanu, A.; Dragoi, B.; Dumitriu, E.; Jerome, F. Selective hydrogenation of furfural to furfuryl alcohol in the presence of a recyclable cobalt/SBA-15 catalyst. ChemSusChem 2015, 8 (11), 18851891.

(24) Sitthisa, S.; An, W.; Resasco, D. E. Selective conversion of furfural to methylfuran over silica-supported $\mathrm{NiFe}$ bimetallic catalysts. J. Catal. 2011, 284, 90-101.

(25) Kijeński, J.; Winiarek, P.; Paryjczak, T. Platinum deposited on monolayer supports in selective hydrogenation of furfural to furfuryl alcohol. Appl. Catal., A 2002, 233, 171-182.

(26) Vargas-Hernández, D.; Rubio-Caballero, J. M.; SantamaríaGonzález, J.; Moreno-Tost, R.; Mérida-Robles, J. M.; Pérez-Cruz, M. A.; Jiménez-López, A.; Hernández-Huesca, R.; Maireles-Torres, P. Furfuryl alcohol from furfural hydrogenation over copper supported on SBA-15 silica catalysts. J. Mol. Catal. A: Chem. 2014, 383-384, $106-113$.

(27) Mironenko, R. M.; Belskaya, O. B.; Gulyaeva, T. I.; Nizovskii, A. I.; Kalinkin, A. V.; Bukhtiyarov, V. I.; Lavrenov, A. V.; Likholobov, V. A. Effect of the nature of carbon support on the formation of active sites in $\mathrm{Pd} / \mathrm{C}$ and $\mathrm{Ru} / \mathrm{C}$ catalysts for hydrogenation of furfural. Catal. Today 2015, 249, 145-152.

(28) Reyes, P.; Salinas, D.; Campos, C.; Oportus, M. Selective hydrogenation of furfural on $\mathrm{Ir} / \mathrm{TiO}_{2}$ catalysts. Quim. Nova 2010, 33, 777-780.

(29) Vetere, V.; Merlo, A. B.; Ruggera, J. F.; Casella, M. L. Transition metal-mased bimetallic catalysts for the chemoselective hydrogenation of furfuraldehyde. J. Braz. Chem. Soc. 2010, 21, 914-920.

(30) Mattson, B.; Foster, W.; Greimann, J.; Hoette, T.; Le, N.; Mirich, A.; Wankum, S.; Cabri, A.; Reichenbacher, C.; Schwanke, E. Heterogeneous catalysis: The Horiuti-Polanyi mechanism and alkene hydrogenation. J. Chem. Educ. 2013, 90, 613-619.

(31) Nakagawa, Y.; Tamura, M.; Tomishige, K. Catalytic conversions of furfural to pentanediols. Catal. Surv. Asia 2015, 19, 249-256.

(32) Shi, Y.; Zhu, Y.; Yang, Y.; Li, Y. W.; Jiao, H. Exploring furfural catalytic conversion on $\mathrm{Cu}(111)$ from computation. ACS Catal. 2015, 5, 4020-4032.

(33) Vorotnikov, V.; Mpourmpakis, G.; Vlachos, D. G. DFT study of furfural conversion to furan, furfuryl alcohol, and 2-methylfuran on $\operatorname{Pd}(111)$. ACS Catal. 2012, 2, 2496-2504.

(34) Wang, S.; Vorotnikov, V.; Sutton, J. E.; Vlachos, D. G. BrønstedEvans-Polanyi and transition state scaling relations of furan derivatives on $\operatorname{Pd}(111)$ and their relation to those of small molecules. ACS Catal. 2014, 4, 604-612.

(35) Wang, S.; Vorotnikov, V.; Vlachos, D. G. Coverage-induced conformational effects on activity and selectivity: hydrogenation and decarbonylation of furfural on $\operatorname{Pd}(111)$. ACS Catal. 2015, 5, 104-112.

(36) Liu, B.; Cheng, L.; Curtiss, L.; Greeley, J. Effects of van der waals density functional corrections on trends in furfural adsorption and hydrogenation on close-packed transition metal surfaces. Surf. Sci. 2014, 622, 51-59.

(37) Williams, R.; Pang, S. H.; Medlin, J. W. Ring opening and oxidation pathways of furanic oxygenates on oxygen-precovered Pd(111). J. Phys. Chem. C 2014, 118, 27933-27943.

(38) Shi, D.; Vohs, J. M. Deoxygenation of biomass-derived oxygenates: reaction of furfural on $\mathrm{Zn}$-modified $\mathrm{Pt}(111)$. ACS Catal. 2015, 5, 2177-2183.

(39) Pang, S. H.; Medlin, J. W. Adsorption and reaction of furfural and furfuryl alcohol on $\operatorname{Pd}(111)$ : unique reaction pathways for multifunctional reagents. ACS Catal. 2011, 1, 1272-1283.

(40) Wilson, K. E.; Baddeley, C. J. Understanding the surface chemistry of enantioselective heterogeneous reactions influence of modification variables on the interaction of methylacetoacetate with 
(S)-aspartic acid modified Ni(111). J. Phys. Chem. C 2009, 113, 10706-10711.

(41) Yao, Y.; Zaera, F. Adsorption and thermal chemistry of formic acid on clean and oxygen-predosed $\mathrm{Cu}(110)$ single-crystal surfaces revisited. Surf. Sci. 2016, 646, 37-44.

(42) Goubert, G.; Groves, M. N.; Dong, Y.; Lemay, J.-C.; McBreen, P. H.; Hammer, B. Isolating a reaction intermediate in the hydrogenation of 2,2,2-trifluoroacetophenone on $\mathrm{Pt}(111)$. J. Phys. Chem. C 2015, 119, 7319-7326.

(43) Lawton, T. J.; Pushkarev, V.; Wei, D.; Lucci, F. R.; Sholl, D. S.; Gellman, A. J.; Sykes, E. C. H. Long range chiral imprinting of $\mathrm{Cu}(110)$ by tartaric acid. J. Phys. Chem. C 2013, 117, 22290-22297. (44) Marcinkowski, M. D.; Liu, J.; Murphy, C. J.; Liriano, M. L.; Wasio, N. A.; Lucci, F. R.; Flytzani-Stephanopoulos, M.; Sykes, E. C. $\mathrm{H}$. Selective formic acid dehydrogenation on $\mathrm{Pt}-\mathrm{Cu}$ single-atom alloys. ACS Catal. 2017, 7, 413-420.

(45) Stacchiola, D.; Calaza, F.; Burkholder, L.; Tysoe, W. T. Vinyl acetate formation by the reaction of ethylene with acetate species on oxygen-covered Pd(111). J. Am. Chem. Soc. 2004, 126, 15384-15385.

(46) Ormerod, R. M.; Baddeley, C. J.; Hardacre, C.; Lambert, R. M. Chemisorption and reactivity of furan on Pd (111). Surf. Sci. 1996, $360,1-9$.

(47) Horcas, I.; Fernández, R.; Gómez-Rodríguez, J. M.; Colchero, J.; Gómez-Herrero, J.; Baro, A. M. WSXM: A software for scanning probe microscopy and a tool for nanotechnology. Rev. Sci. Instrum. 2007, 78, 013705-013712.

(48) Lavoie, S.; McBreen, P. H. Evidence for $\mathrm{C}-\mathrm{H} \cdots \mathrm{O}=\mathrm{C}$ bonding in coadsorbed aromatic-carbonyl systems on $\mathrm{Pt}(111)$. J. Phys. Chem. B 2005, 109, 11986-11990.

(49) Redhead, P. A. Thermal desorption of gases. Vacuum 1962, 12 (4), 203-211.

(50) Ihm, H.; White, J. M. Stepwise dissociation of thermally activated phenol on $\mathrm{Pt}(111)$. J. Phys. Chem. B 2000, 104, 6202-6211.

(51) Ihm, H.; Ajo, H. M.; Gottfried, J. M.; Bera, P.; Campbell, C. T. Calorimetric measurement of the heat of adsorption of benzene on Pt(111). J. Phys. Chem. B 2004, 108, 14627-14633.

(52) Gottfried, J. M.; Vestergaard, E. K.; Bera, P.; Campbell, C. T. Heat of adsorption of naphthalene on $\mathrm{Pt}(111)$ measured by adsorption calorimetry. J. Phys. Chem. B 2006, 110, 17539-17545.

(53) Avery, N. R. Adsorption and reactivity of cyclopentane on $\operatorname{Pt}(111)$. Surf. Sci. 1985, 163, 357-368.

(54) Liu, B.; Cheng, L.; Curtiss, L.; Greeley, J. Effects of van der waals density functional corrections on trends in furfural adsorption and hydrogenation on close-packed transition metal surfaces. Surf. Sci. 2014, 622, 51-59.

(55) Rogers, S. M.; Catlow, C. R. A.; Chan-Thaw, C. E.; Chutia, A.; Jian, N.; Palmer, R. E.; Perdjon, M.; Thetford, A.; Dimitratos, N.; Villa, A.; et al. Tandem site and size controlled Pd nanoparticles for the directed hydrogenation of furfural. ACS Catal. 2017, 7, 2266-2274.

(56) Baker, L. R.; Kennedy, G.; Van Spronsen, M.; Hervier, A.; Cai, X.; Chen, S.; Wang, L.-W.; Somorjai, G. A. Furfuraldehyde hydrogenation on titanium oxide-supported platinum nanoparticles studied by sum frequency generation vibrational spectroscopy: acidbase catalysis explains the molecular origin of strong metal-support interactions. J. Am. Chem. Soc. 2012, 134, 14208-14216.

(57) Gebhard, S. C.; Koel, B. E. Influence of potassium on the adsorption of hydrogen on platinum(111). J. Phys. Chem. 1992, 96, $7056-7063$.

(58) Christmann, K.; Ertl, G.; Pignet, T. Adsorption of hydrogen on a $\mathrm{Pt}(111)$ surface. Surf. Sci. 1976, 54, 365-392.

(59) An, K.; Musselwhite, N.; Kennedy, G.; Pushkarev, V. V.; Baker, L. R.; Somorjai, G. A. Preparation of mesoporous oxides and their support effects on Pt nanoparticle catalysts in catalytic hydrogenation of furfural. J. Colloid Interface Sci. 2013, 392 (392), 122-128. 